\title{
Longitudinal patterns of bone-targeted agent use among patients with solid tumors and bone metastases in the United States
}

\author{
Yi Qian ${ }^{1}$ - Debajyoti Bhowmik ${ }^{1}$ - Nandita Kachru ${ }^{2}$ - Rohini K. Hernandez ${ }^{3}$
}

Received: 30 March 2016 / Accepted: 9 January 2017 / Published online: 24 January 2017

(C) The Author(s) 2017. This article is published with open access at Springerlink.com

\begin{abstract}
Purpose This study examined real-world long-term use of guideline-recommended bone targeted agents (BTA) among patients with metastatic solid tumors.

Methods Adults with a solid tumor diagnosis followed by a bone metastasis diagnosis in 2012-2014 were identified from electronic medical records in the Oncology Services Comprehensive Electronic Records (OSCER) database. Patients initiated zoledronic acid (ZA) or denosumab on or after the bone metastasis diagnosis and were followed through last clinic visit by 30 June 2015 . We describe time to BTA initiation, compliance ( $\geq 12$ administrations in a year), switching, and non-persistence (switch or $\geq 90$ day gap in therapy), by agent and follow-up period.

Results The majority of the 14,881 study patients $(50 \%$ female, $65 \%$ age $\geq 65$ years) had breast ( $33 \%$ ), prostate ( $26 \%$ ), or lung (26\%) tumors. Half of all patients initiated on each agent, with denosumab initiations exceeding ZA initiations in 2014. Most (91\% denosumab, 93\% ZA) initiations occurred within 3 months of bone metastasis diagnosis. At 1, 2, and 3 years post-initiation, denosumab patients were less likely to
\end{abstract}

Electronic supplementary material The online version of this article (doi:10.1007/s00520-017-3583-1) contains supplementary material, which is available to authorized users.

Yi Qian

yiq@amgen.com

1 Global Health Economics, Amgen Inc., One Amgen Center Drive, M/S 28-3-A, Thousand Oaks, CA 91320-1799, USA

2 Department of Pharmaceutical Health Outcomes and Policy, College of Pharmacy, University of Houston, Houston, TX, USA

3 Center for Observational Research, Amgen Inc., Thousand Oaks, CA, USA switch agents $(4,3$, and $1 \%$ versus 14,12 , and $11 \%)$ and more likely to be compliant $(50,37$, and $31 \%$ versus 41,26 , and $6 \%$ ). Median time to non-persistence was 25.9 months for denosumab and 17.2 months for ZA, $p<0.0001$.

Conclusions This is the first study reporting long-term treatment patterns for the two primary BTAs used in the USA. The greater compliance and longer persistence observed among denosumab patients may improve treatment effectiveness achieved in the real-world setting.

Keywords Bone metastasis $\cdot$ Solid tumor $\cdot$ Bone targeted agents $\cdot$ Persistence $\cdot$ Compliance

\section{Introduction}

Bone metastases are a common outcome in patients with solid tumors and are present in up to $70 \%$ of patients with advanced breast and prostate cancer and more than one third of patients with advanced lung cancer [1]. Bone metastases are clinically important because they increase the risk for a variety of debilitating skeletal-related events (SREs), including spinal cord compression, bone surgery, pathologic fractures, and severe bone pain requiring palliative radiotherapy $[2,1]$. These events are common with observational studies reporting a 1year incidence of SREs at $46 \%$ in prostate cancer patients and $38 \%$ in female breast cancer patients $[3,4]$ and clinical trials reporting a 2-year cumulative incidence of $49 \%$ in prostate cancer patients and $68 \%$ in breast cancer patients who were not receiving treatment for bone metastases [5, 6]. Experience in routine clinical practice (January 1995 to December 2009) in two large health systems in the United States (US) suggests that 63,59 , and $52 \%$ of breast, lung, and prostate cancer patients, respectively, experience an SRE either at diagnosis or 
during follow-up (median follow-up months: 13.6, 3.1, 16.6) [7].

SREs that occur subsequent to bone metastases in patients with solid tumors are associated with shortened survival [3, 4]. In addition, patients report poorer health status and lower health-related quality of life following an SRE, and these events impose a significant economic burden on patients and the health care system overall through increased use of health services including surgical interventions, as well as office visits and medications to address SRE-related pain $[8,9]$.

Over the last decade, bone health has become an important component of guideline-recommended care for patients with solid tumors [10-12]. Since 1996, the US Food and Drug Administration (FDA) has approved three bone targeted agents (BTA) for use in prevention of SREs in patients with bone metastasis secondary to solid tumors. These include zoledronic acid (4 mg) and pamidronate disodium [13], both of which are intravenous bisphosphonates (IVBP) with dosing every 3-4 weeks and denosumab (120 mg), a RANK ligand inhibitor [14] with dosing every 4 weeks. With its FDA approval in 2002, zoledronic acid became the predominant IVBP for treatment of bone metastasis in the US, in part because it offers a shorter infusion time (a minimum of $15 \mathrm{~min}$ ) compared with that of pamidronate which requires at least 2 hours [15]. In 2010, the treatment landscape changed again when the FDA approved denosumab for prevention of skeletal-related events in patients with solid tumors. As a first-in-class RANK ligand inhibitor, this agent offers a more efficient, subcutaneous mode of administration which appeals to patients as well as physicians $[16,17]$.

These agents represent important treatment options and are included in clinical guidelines for patients with advanced solid tumors; yet, there is limited literature describing their use in routine practice in the US [18-20,10]. The most recent data from these studies report the experience of patients who initiated BTA treatment through 2011, with follow-up periods of up to 12 months. Changes in the cancer treatment landscape are leading to increased survival even among patients with advanced cancer, and extended use of supportive care is likely to be more common. The current study examines BTA use among patients with metastatic solid tumors who initiated a BTA between 2012 and 2014.

\section{Methods}

This study used data from the Oncology Services Comprehensive Electronic Records (OSCER) database which includes electronic medical records (EMRs) from $>750,000$ cancer patients treated at $>500$ US community and hospitalaffiliated oncology clinics, since 2004. These clinical practices include all payer types (commercial, Medicare, Medicaid, other, and cash) and patients residing in all 50 states. At each patient visit, data on diagnoses and treatments including outpatient chemotherapy and BTA administrations are captured in the EMR and entered in to the database.

From the OSCER database, patients with a bone metastasis diagnosis (International Classification of Disease Coding Manual [ICD-9-CM] code 198.5) between 1 January 2012 and 31 December 2014 were identified. Included patients were also required to have evidence of a solid tumor, excluding multiple myeloma, as indicated by the presence of at least one record with a relevant ICD-9$\mathrm{CM}$ diagnosis code on or prior to first identified bone metastasis diagnosis. Patients were also required to be 18 years of age or older as of the bone metastasis diagnosis. This study focuses on patients who used either zoledronic acid or denosumab since these are the predominant BTAs in current use in the US. Patients in the study population received their first administration of either zoledronic acid or denosumab on or after the bone metastasis diagnosis with a lookback period of 6 months to ensure that they were incident BTA users. Compliance and persistence outcomes were assessed during the first, second, and third year after BTA initiation, among patients with data available for the entirety of each of these follow-up periods.

Demographics (age, sex, race/ethnicity) and disease characteristics (location of primary solid tumor, time since cancer diagnosis, time since bone metastases diagnosis) of study patients were captured as of the date patients initiated BTA (index date). Patients were followed for up to 36 months after BTA initiation, with follow-up censored at the last clinic visit recorded in OSCER by 30 June 2015. The average number of BTA administrations and percentage of patients who received at least 12 administrations in a year were determined for patients with follow-up in each of the three 1-year intervals encompassed in the study period. This latter measure serves as a proxy for compliance given the expectation that administrations should occur in 3-4-week intervals. Switching between agents was also assessed for patients with follow-up in each of the three 1-year intervals. Non-persistence with BTA therapy was assessed over 36 months of follow-up using a previously published definition [21] of a gap in therapy of 90 days or longer, or 90 days or longer gap between the last dose and the end of follow-up, or switch from the initial agent. Sensitivity analyses were also performed using 60- and 120day therapy gaps to define non-persistence. Kaplan-Meier analysis was used to describe the probability of nonpersistence during follow-up, and time to non-persistence was defined as the time from the index date to the date of first occurrence of non-persistence. Statistical analysis was performed using SAS® software for Windows, version 9.2 (SAS Institute Inc., Cary, North Carolina). Test statistics ( $P$ values) were generated using $t$ tests for continuous variables and Chi-square tests for categorical variables; no adjustments were made for multiplicity. 


\section{Results}

The study included 14,881 patients (Supplemental Fig. 1) who met all selection criteria. Of these, $50 \%$ were female and $65 \%$ were aged 65 years or older (Table 1). The majority of patients had breast (33\%), prostate (26\%), or lung $(26 \%)$ tumors. The number of patients included in the study increased each year from 4106 (28\%) in 2012 to 5059 (34\%) in 2013 and to 5716 $(38 \%)$ in 2014. Overall, approximately half of study patients initiated on denosumab (49\%) and half $(51 \%)$ on zoledronic acid. The percentage of patients who initiated on denosumab increased over the study period and, in 2014, exceeded that of patients initiating zoledronic acid (Fig. 1).

The demographic and clinical characteristics of patients who initiated denosumab differed from those of patients who initiated zoledronic acid (Table 1). Denosumab patients were more likely to be older and also more likely to be male, which reflects the fact that patients with prostate cancer comprised a larger proportion of the denosumab patient group than of the zoledronic acid patient group (30.4\% versus $21.0 \%$, $p<0.0001)$. Patients with breast cancer comprised a similar proportion of the patients using each agent $(p=0.39)$. Patients with lung cancer and those with other types of solid tumors (i.e., non-breast, non-prostate, non-lung) represented a smaller

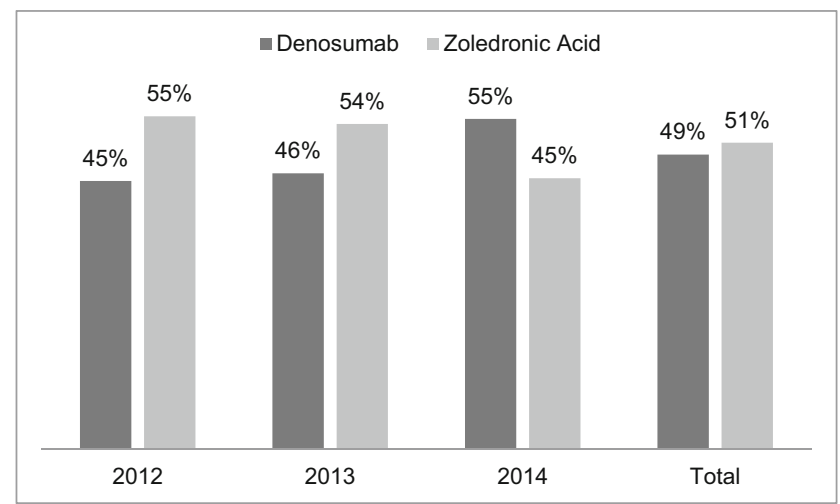

BTA: bone-targeted agent

Fig. 1 BTA initiations by agent and year

proportion of denosumab patients than of zoledronic acid patients $(22.3 \%$ versus $29.4 \%, p<0.0001 ; 14.4 \%$ versus $15.9 \%$, $p=0.01)$.

The distribution of patients by time between cancer diagnosis and BTA initiation differed between the denosumab and zoledronic acid patients, although the magnitude of these differences was small (Table 1). This was also the case for the time between bone metastasis diagnosis and BTA initiation. Overall, the majority (92\%) of patients initiated BTA within

Table 1 Characteristics of patients with bone metastases secondary to solid tumors who initiated a bone-targeted agent in 2012-2014

\begin{tabular}{|c|c|c|c|c|}
\hline & All patients $(N=14,881)$ & $\begin{array}{l}\text { Denosumab } \\
(N=7294 / 49 \%)\end{array}$ & $\begin{array}{l}\text { Zoledronic acid } \\
(N=7587 / 51 \%)\end{array}$ & $P$ value \\
\hline \multicolumn{5}{|l|}{ Baseline demographics } \\
\hline Male & $7407(49.8)$ & $3804(52.2)$ & $3603(47.5)$ & $<0.0001$ \\
\hline Race/ethnicity: Asian & $192(1.5)$ & $78(1.2)$ & $114(1.7)$ & $<0.0001$ \\
\hline African American & $939(7.2)$ & $414(6.5)$ & $525(7.9)$ & \\
\hline Other race & $1397(10.8)$ & $583(9.2)$ & $814(12.3)$ & \\
\hline White & $10,445(80.5)$ & $5283(83.1)$ & $5162(78.0)$ & \\
\hline \multicolumn{5}{|l|}{ Tumor characteristics } \\
\hline \multicolumn{5}{|l|}{ Location of primary tumor } \\
\hline Breast & $4914(33)$ & $2384(32.6)$ & $2530(33.4)$ & 0.39 \\
\hline Prostate & $3816(25.6)$ & $2220(30.4)$ & $1596(21)$ & $<0.0001$ \\
\hline Lung & $3856(25.9)$ & $1626(22.3)$ & $2230(29.4)$ & $<0.0001$ \\
\hline Other solid tumors & $2251(15.1)$ & $1048(14.4)$ & $1203(15.9)$ & 0.01 \\
\hline Multiple primary tumors & $44(0.30)$ & $16(0.22)$ & $28(0.36)$ & - \\
\hline \multicolumn{5}{|c|}{ Time from cancer diagnosis to BTA initiation } \\
\hline$<1$ year & $9897(66.5)$ & $4766(65.3)$ & $5131(67.6)$ & 0.01 \\
\hline $1-2$ years & $1420(9.5)$ & $710(9.7)$ & $710(9.4)$ & \\
\hline$\geq 2$ years & $3564(24)$ & $1818(24.9)$ & $1746(23.0)$ & \\
\hline \multicolumn{5}{|c|}{ Time from bone metastasis diagnosis to BTA initiation } \\
\hline$<1$ year & $14,582(98)$ & $7125(97.7)$ & $7457(98.3)$ & 0.02 \\
\hline $1-2$ years & $261(1.8)$ & $150(2.06)$ & $111(1.5)$ & \\
\hline$\geq 2$ years & $38(0.3)$ & $19(0.26)$ & $19(0.2)$ & \\
\hline
\end{tabular}

${ }^{\mathrm{a}}$ Does not sum up to $N=14,881$ due to missing values 
3 months of the bone metastasis diagnosis. Ninety-one percent of denosumab patients initiated within 3 months compared with $93 \%$ of zoledronic acid patients, and the percentage of patients initiating within 6 months was similar between the two patient groups (95 and 96\%, respectively).

The average number of BTA administrations per patient overall was highest in the first year of follow-up and higher in all follow-up periods for denosumab patients compared with patients treated with zoledronic acid (Fig. 2). Patients treated with denosumab were also more likely to remain compliant, that is to receive at least 12 administrations in a 1-year period (Fig. 3). When results were analyzed by tumor type, treatment initiation and use patterns were similar to those for the full solid tumor population.

Patients using denosumab were also less likely to switch to zoledronic acid than vice versa (Fig. 4). In the first year of follow-up, the percentage of patients switching agents was $4 \%$ for denosumab patients versus $14 \%$ for patients using zoledronic acid. In the second and third years, 3 and $1 \%$ of denosumab patients switched compared with 12 and $11 \%$ of patients using zoledronic acid. Among patients using denosumab, 77, 54, and $38 \%$ were persistent at 12,24 , and 36 months of follow-up (Fig. 5). By comparison, persistence among patients using zoledronic acid was 64,36 , and $23 \%$ in each of these intervals. Regardless of the therapy gap used (60, 90, 120 days), persistence was higher among patients using denosumab than among patients using zoledronic acid. Using the 90-day therapy gap, the median time to nonpersistence over the entire 36-month follow-up was 25.9 months for denosumab and 17.2 months for zoledronic acid, $p<0.0001$.

\section{Discussion}

This study updates previous studies of real-world BTA treatment patterns among advanced solid tumor patients with more

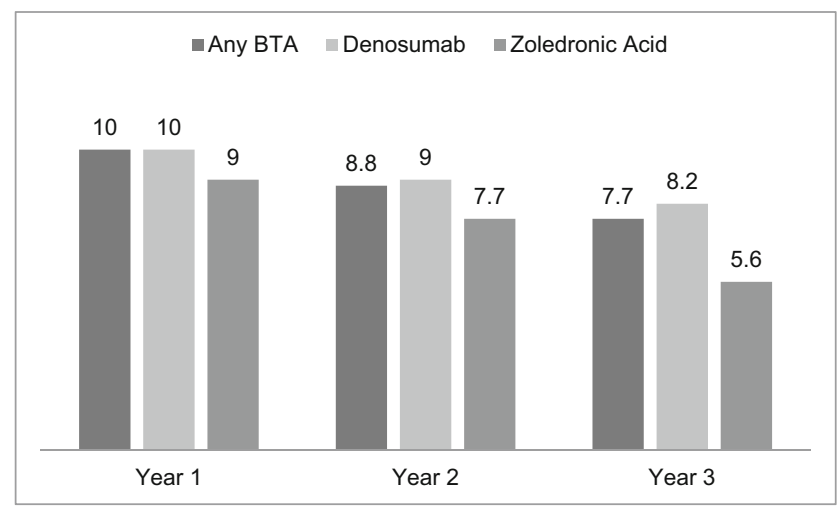

BTA: bone-targeted agent

Fig. 2 Average number of BTA administrations by agent and follow-up period

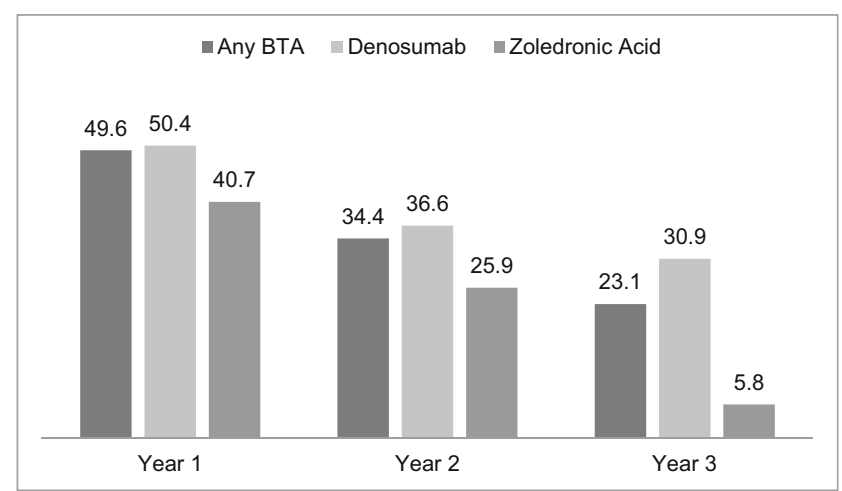

BTA: bone-targeted agent

${ }^{\mathrm{a}} \geq 12$ administrations/ year

Fig. 3 Percent of compliant ${ }^{\mathrm{a}}$ patients by agent and follow-up period

recent data (BTA initiations between 1 January 2012 and 31 December 2014) and provides a long-term perspective by examining utilization in 1-year intervals for up to 3 years of follow-up after BTA initiation. Thus, we aimed to reflect utilization in current practice at US oncology clinics, in the period during which physicians and patients have gained significant experience with the most recently approved BTA, denosumab.

Although two recent administrative claim based studies have examined treatment patterns for IV BP in solid tumor patients beyond 1 year $[19,20]$, the only study to include all available treatment options reported BTA treatment patterns observed in the Oncology Services Comprehensive Electronic Records (OSCER) for patients with solid tumors whose first bone metastasis was diagnosed in 2011, with a maximum of 12-month follow-up [18]. The current study adds to these previously reported results by examining BTA treatment patterns for the two most commonly used agents, denosumab and zoledronic acid, over up to 3 years of follow-up.

Results of the current study indicate that the majority of solid tumor patients who initiate BTA therapy do so soon after

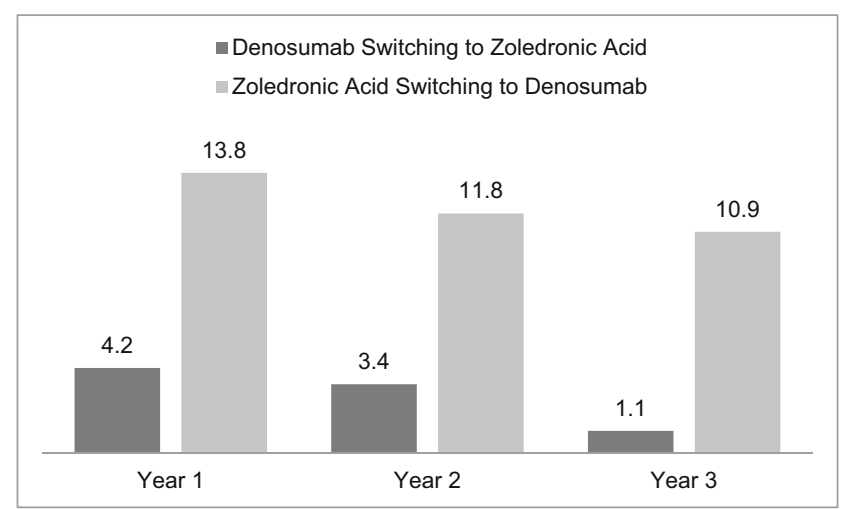

BTA: bone-targeted agent

Fig. 4 Percent of patients switching BTA by index drug and follow-up period 


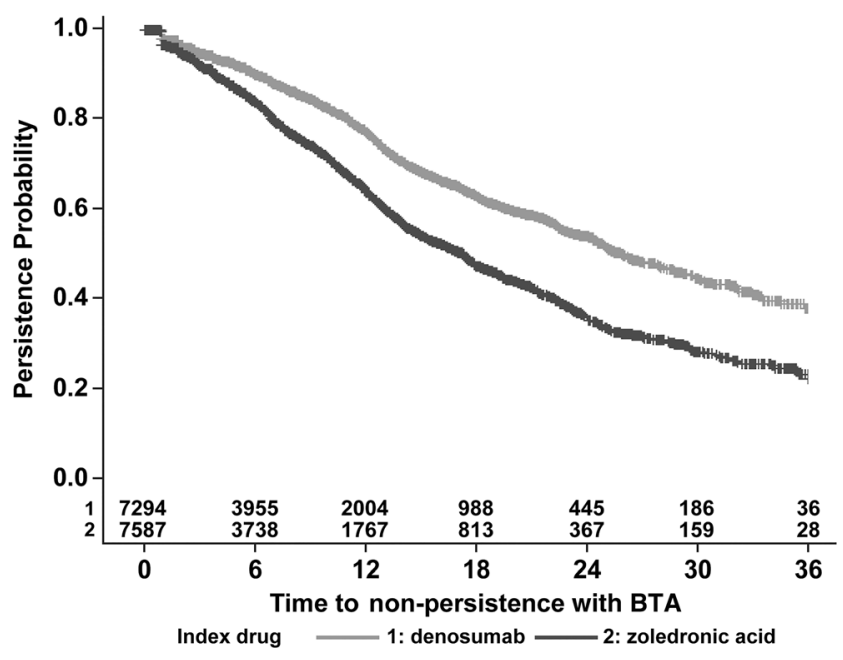

Fig. 5 Time to non-persistence with BTA

bone metastasis diagnosis which is consistent with previously reported findings $[20,18]$. However, this finding should be interpreted with caution. As Oster et al. note, SREs may be the catalyst for the initiation of BTA therapy, since in that study, similar percentages of breast cancer and prostate cancer patients initiated IV BP prior to and after experiencing their first skeletal complication [19]. Furthermore, the recording of bone metastasis diagnosis may commonly be delayed until treatment is initiated. In a validation study of the OSCER data among breast cancer patients, a median of 32 days was found in between the true date of bone metastasis diagnosis (per patient chart), and the date diagnosis was coded in the electronic medical record data, with a median delay of 43 days observed for administrative claim data [22]. This delayed reporting would therefore cause an underestimation in the time between initial diagnosis and treatment initiation.

Characteristics of patients who initiated on denosumab differed in some respects from those of patients who initiated on zoledronic acid. Compared with patients initiating zoledronic acid, denosumab patients were older. Although our study did not examine treatment initiation rates, previous studies have reported that the IV BP initiation among patients with bone metastases varies with age and is significantly lower among patients aged 65 and older compared with younger patients $[20,19]$. Additional research would need to be undertaken to determine if the approval of denosumab has improved the bone metastasis treatment rate among older patients since the current study does not provide insights into whether treatment rates in this important patient subgroup are changing over time.

Compliance and persistence are important dimensions of medication use and shape the therapeutic benefit that patients may achieve. For our study, compliance was defined as 12 or more administrations in a 12-month period, although compliance to a strict 4-week dosing schedule would yield 13 administrations in a year. In each of the three 1-year periods evaluated, a greater percentage of denosumab patients were compliant compared with zoledronic acid users. In the third year of follow-up, compliance exceeded $30 \%$ among denosumab patients in contrast to $6 \%$ among patients using zoledronic acid. This consistently greater compliance observed throughout the 3 -year study period confirms and extends the 1-year findings reported by Hernandez et al. As suggested previously, the better compliance among denosumab patients may reflect more convenient dosing and physicians' motivation to sustain "on-time" dosing schedules in order to achieve the full therapeutic benefit demonstrated in the denosumab clinical trials. Recent research into physician preferences for bone metastasis drug therapy indicates that the most important considerations are delaying SREs and worsening of pain, while minimizing the risk of renal impairment $[16,23]$. Renal toxicity is also an important factor when patients consider BTA therapy, and the known renal toxicity of IV BPs complicates treatment since renal function must be closely monitored in patients using zoledronic acid and doses may be delayed or skipped depending on the patient serum creatinine levels $[16,24,25]$. That level of monitoring is not required for denosumab which may also make compliance with denosumab easier to achieve, particularly among patients with complicated regimens of oncologic and supportive therapies, regardless of age.

In addition to demonstrating higher compliance, denosumab patients in our study also remained persistent with therapy longer than patients using zoledronic acid (median time to nonpersistence: 25.9 months versus 17.2 , $p<0.0001)$. The persistence rates for denosumab were 77 , 54 , and $38 \%$, while persistence rates for zoledronic acid were 64,36 , and $23 \%$, at 12,24 , and 36 months of follow-up, respectively. Other researchers have also shown that persistence is challenging for patients using IV BP. Hagiwara et al., for example, estimated that $46 \%$ of breast cancer patients, $56 \%$ of prostate cancer patients, and $83 \%$ of lung cancer patients had discontinued IVBP therapy at 12 months after IV BP initiation [20].

Outside of clinical trials, treatment persistence with BTA may vary, and several retrospective studies of administrative claim data have documented better treatment effectiveness for IV BP among patients with longer persistence, although comparison of results is challenging since definitions of non-persistence vary. In one such study in which non-persistence was defined as a therapy gap of more than 45 days, persistent use of monthly zoledronic acid for 18 months was associated with a $50 \%$ lower risk of an SRE and a $63 \%$ lower risk of a fracture compared with persistent monthly use for less than 3 months among breast cancer patients [26]. In two other 
studies, zoledronic acid patients were classified as having short ( $\leq 90$ days), medium (91-180 days), or long ( $>180$ days) persistence with non-persistence defined by a therapy gap of $>45$ days $[27,28]$. Among breast cancer patients, those with long persistence had significantly lower risk of skeletal complications, although there were no significant differences between the short and medium persistence groups [28]. Among prostate cancer patients, however, there were no significant differences in the risk of skeletal complications between persistence groups, although more persistent patients had significantly longer follow-up durations [27]. An earlier study by Hatoum et al. demonstrated that in both 6 and 12 months of follow-up, the rate of skeletal-related complications was significantly lower in patients who received zoledronic acid administrations according to the recommended dosing schedule compared with patients who underwent less frequent dosing [29]. There are currently no data available describing the influence of compliance and persistence on the real-world effectiveness of denosumab.

This study presents unique insights into the long-term treatment patterns for the two most commonly used BTAs. As with all studies, however, there are important limitations. First, the study population includes only patients who were treated at an oncology clinic which contributes data to the OSCER database. Patients who moved or sought care outside of these clinics would be lost to follow-up and no longer contribute data to this data source. As noted previously, time between bone metastasis diagnosis and treatment initiation may be underestimated since the occurrence of an SRE or BTA initiation may trigger the recording of a bone metastasis diagnosis and there are likely substantial delays between the documentation of a bone metastasis diagnosis in the patient's medical chart and the appearance of that diagnosis in either the EMR or on an administrative claim record. The Kaplan-Meier analysis used to assess persistence does take follow-up time into consideration. However, mortality data were not available in the source data, and therefore, the relationship between survival and persistence could not be directly assessed.

\section{Conclusions}

This study is the first to examine long-term treatment patterns for the two primary BTAs used in the US. In each of the three 1 -year periods evaluated, a greater percentage of denosumab patients were compliant compared with zoledronic acid users. Denosumab patients also had longer durations of persistent therapy use. These higher levels of compliance and persistence may improve treatment effectiveness achieved in this real-world setting.

\section{Compliance with ethical standards}

Conflicts of interest This study was funded by Amgen Inc., Thousand Oaks, CA. YQ, DB, and RKH are employees and stockholders of Amgen Inc. and have access to the primary study data. NK was employed by Amgen Inc. at the time of the study.

Open Access This article is distributed under the terms of the Creative Commons Attribution-NonCommercial 4.0 International License (http:// creativecommons.org/licenses/by-nc/4.0/), which permits any noncommercial use, distribution, and reproduction in any medium, provided you give appropriate credit to the original author(s) and the source, provide a link to the Creative Commons license, and indicate if changes were made.

\section{References}

1. Coleman RE (2006) Clinical features of metastatic bone disease and risk of skeletal morbidity. Clinical cancer research: an official journal of the American Association for Cancer Research 12(20 Pt 2): 6243s-6249s. doi:10.1158/1078-0432.CCR-06-0931

2. Coleman RE (2004) Bisphosphonates: clinical experience. Oncologist 9(Suppl 4):14-27. doi:10.1634/theoncologist.990004-14

3. Norgaard M, Jensen AO, Jacobsen JB, Cetin K, Fryzek JP, Sorensen HT (2010) Skeletal related events, bone metastasis and survival of prostate cancer: a population based cohort study in Denmark (1999 to 2007). J Urol 184(1):162-167. doi:10.1016/j. juro.2010.03.034

4. Yong M, Jensen AO, Jacobsen JB, Norgaard M, Fryzek JP, Sorensen HT (2011) Survival in breast cancer patients with bone metastases and skeletal-related events: a population-based cohort study in Denmark (1999-2007). Breast Cancer Res Treat 129(2): 495-503. doi:10.1007/s10549-011-1475-5

5. Lipton A, Theriault RL, Hortobagyi GN, Simeone J, Knight RD, Mellars K, Reitsma DJ, Heffernan M, Seaman JJ (2000) Pamidronate prevents skeletal complications and is effective palliative treatment in women with breast carcinoma and osteolytic bone metastases: long term follow-up of two randomized, placebocontrolled trials. Cancer 88(5):1082-1090

6. Saad F, McKiernan J, Eastham J (2006) Rationale for zoledronic acid therapy in men with hormone-sensitive prostate cancer with or without bone metastasis. Urol Oncol 24(1):4-12. doi:10.1016/j. urolonc.2005.06.020

7. Oster G, Lamerato L, Glass AG, Richert-Boe KE, Lopez A, Chung K, Richhariya A, Dodge T, Wolff GG, Balakumaran A, Edelsberg J (2013) Natural history of skeletal-related events in patients with breast, lung, or prostate cancer and metastases to bone: a 15-year study in two large US health systems. Supportive care in cancer: official journal of the Multinational Association of Supportive Care in Cancer 21(12):3279-3286. doi:10.1007/s00520-013-1887-3

8. Schulman KL, Kohles J (2007) Economic burden of metastatic bone disease in the U.S. Cancer 109(11):2334-2342. doi:10.1002/cncr.22678

9. Weinfurt KP, Castel LD, Li Y, Timbie JW, Glendenning GA, Schulman KA (2004) Health-related quality of life among patients with breast cancer receiving zoledronic acid or pamidronate disodium for metastatic bone lesions. Med Care 42(2):164-175. doi:10.1097/01.mlr.0000108746.69256.45

10. Coleman R, Body JJ, Aapro M, Hadji P, Herrstedt J, Group EGW (2014) Bone health in cancer patients: ESMO clinical practice 
guidelines. Annals of oncology: official journal of the European Society for Medical Oncology / ESMO 25(Suppl 3):iii124-iii137. doi:10.1093/annonc/mdu103

11. Gralow JR, Biermann JS, Farooki A, Fornier MN, Gagel RF, Kumar R, Litsas G, McKay R, Podoloff DA, Srinivas S, Van Poznak CH (2013) NCCN task force report: bone health in cancer care. Journal of the National Comprehensive Cancer Network: JNCCN 11(Suppl 3):S1-50 quiz S51

12. National Comprehensive Cancer Network. Clinical practice guidelines. http://www.ncen.org/professionals/physician gls/f guidelines.asp\#site. Accessed 23 Feb 2016

13. Aredia (pamidronate disodium) prescribing information. http://www.novartis.com.au/PI PDF/apd.pdf. Accessed 31 Jan 2016

14. XGEVA (denosumab) prescribing information. http://www.xgeva. com/hcp/xgeva-dosing-and-administration.html. Accessed 31 Jan 2016

15. Body JJ (2012) Denosumab for the management of bone disease in patients with solid tumors. Expert Rev Anticancer Ther 12(3):307322. doi:10.1586/era.11.204

16. Arellano J, Gonzalez JM, Qian Y, Habib M, Mohamed AF, Gatta F, Hauber AB, Posner J, Califaretti N, Chow E (2015) Physician preferences for bone metastasis drug therapy in Canada. Curr Oncol 22(5):e342-e348. doi:10.3747/co.22.2380

17. Hauber AB, Arellano J, Qian Y, Gonzalez JM, Posner JD, Mohamed AF, Gatta F, Tombal B, Body JJ (2014) Patient preferences for treatments to delay bone metastases. Prostate 74(15): 1488-1497. doi:10.1002/pros.22865

18. Hernandez RK, Quigley J, Pirolli M, Quach D, Chen KS, Arellano J, Liede A (2014) Patients with bone metastases from solid tumors initiating treatment with a bone-targeted agent in 2011: a descriptive analysis using oncology clinic data in the US. Supportive care in cancer: official journal of the Multinational Association of Supportive Care in Cancer 22(10):2697-2705. doi:10.1007/s00520-014-2251-y

19. Oster G, Lamerato L, Glass AG, Richert-Boe KE, Lopez A, Chung K, Richhariya A, Dodge T, Wolff GG, Balakumaran A, Edelsberg J (2014) Use of intravenous bisphosphonates in patients with breast, lung, or prostate cancer and metastases to bone: a 15-year study in two large US health systems. Supportive care in cancer: official journal of the Multinational Association of Supportive Care in Cancer 22(5):1363-1373. doi:10.1007/s00520-013-2094-y

20. Hagiwara M, Delea TE, Cong Z, Chung K (2014) Utilization of intravenous bisphosphonates in patients with bone metastases secondary to breast, lung, or prostate cancer. Supportive care in cancer: official journal of the Multinational Association of Supportive Care in Cancer 22(1):103-113. doi:10.1007/s00520-013-1951-z

21. Hernandez RK, Adhia A, Wade SW, O'Connor E, Arellano J, Francis K, Alvrtsyan H, Million RP, Liede A (2015) Prevalence of bone metastases and bone-targeting agent use among solid tumor patients in the United States. Clinical epidemiology 7:335-345. doi:10.2147/CLEP.S85496

22. Liede A, Hernandez RK, Roth M, Calkins G, Larrabee K, Nicacio L (2015) Validation of international classification of diseases coding for bone metastases in electronic health records using technology-enabled abstraction. Clinical epidemiology 7:441-448. doi:10.2147/CLEP.S92209

23. Arellano J, Hauber AB, Mohamed AF, Gonzalez JM, Collins H, Hechmati G, Gatta F, Qian Y (2015) Physicians' preferences for bone metastases drug therapy in the United States. Value in health: the journal of the International Society for Pharmacoeconomics and Outcomes Research 18(1):78-83. doi:10.1016/j.jval.2014.10.004

24. Hechmati G, Hauber AB, Arellano J, Mohamed AF, Qian Y, Gatta F, Haynes I, Bahl A, von Moos R, Body JJ (2015) Patients' preferences for bone metastases treatments in France, Germany and the United Kingdom. Supportive care in cancer: official journal of the Multinational Association of Supportive Care in Cancer 23(1):2128. doi:10.1007/s00520-014-2309-x

25. Mortimer JE, Pal SK (2010) Safety considerations for use of bonetargeted agents in patients with cancer. Semin Oncol 37(Suppl 1): S66-S72. doi:10.1053/j.seminoncol.2010.06.006

26. Henk HJ, Kaura S (2012) Retrospective database analysis of the effect of zoledronic acid on skeletal-related events and mortality in women with breast cancer and bone metastasis in a managed care plan. J Med Econ 15(1):175-184. doi:10.3111/13696998.2011.632044

27. Hatoum HT, Lin SJ, Guo A, Lipton A, Smith MR (2011) Zoledronic acid therapy impacts risk and frequency of skeletal complications and follow-up duration in prostate cancer patients with bone metastasis. Curr Med Res Opin 27(1):55-62. doi:10.1185/03007995.2010.535511

28. Hatoum HT, Lin SJ, Smith MR, Guo A, Lipton A (2011) Treatment persistence with monthly zoledronic acid is associated with lower risk and frequency of skeletal complications in patients with breast cancer and bone metastasis. Clinical breast cancer 11(3):177-183 . doi:10.1016/j.clbc.2011.03.015

29. Hatoum HT, Lin SJ, Smith MR, Barghout V, Lipton A (2008) Zoledronic acid and skeletal complications in patients with solid tumors and bone metastases: analysis of a national medical claims database. Cancer 113(6):1438-1445. doi:10.1002/cncr.23775 